\title{
Tradução, adaptação transcultural para o português (Brasil) e validação de conteúdo da Body Checking Cognitions Scale (BCCS)
}

\author{
Portuguese (Brazil) translation, cross-cultural adaptation and content validity of Body Checking \\ Cognitions Scale-BCCS
}

\author{
Adriana Trejger Kachani ${ }^{1}$ Ana Lucia Rodrigues Barbosa², Silvia Brasiliano ${ }^{3}$, Táki Athanássios Cordás4, \\ Patrícia Brunfentrinker Hochgraf5, Maria Aparecida Conti ${ }^{6}$
}

\author{
${ }^{\top}$ Nutricionista responsável pelo Programa de Atenção à Mulher Dependente Química (Promud) do Instituto de Psiquiatria do Hospital das Clínicas da Faculdade de Medicina da Universidade de \\ São Paulo (IPq-HCFMUSP), doutoranda pela FMUSP. \\ 2 Nutricionista do Promud/IPq-HCFMUSP. \\ ${ }^{3}$ Coordenadora-executiva do Promud/IPq-HCFMUSP, doutora em Ciências pela FMUSP. \\ ${ }_{4}^{4}$ Coordenador-geral do Ambulatório de Bulimia e Transtornos Alimentares (Ambulim) do IPq-HCFMUSP, professor-colaborador do Departamento de Psiquiatria da FMUSP, professor do Programa de \\ Pós-Graduação do Departamento de Psiquiatria da FMUSP e do programa de Neurociências e Comportamento do Instituto de Psicologia da USP. \\ 5 Coordenadora clínica do Promud/IPq-HCFMUSP, doutora em Psiquiatria pela FMUSP. \\ ${ }^{6}$ Doutora em Saúde Pública pela Faculdade de Saúde Pública (FSP) da USP, pesquisadora e docente do Ambulim/IPq-HCFMUSP.
}

Recebido: 4/11/2009 - Aceito: 31/3/2010

\section{Resumo}

Contexto: A Body Checking Cognitions Scale (BCCS) avalia cognições relacionadas à checagem do peso, comportamento comum e de importância clínica em pacientes com transtornos alimentares (TA). Objetivo: Tradução, adaptação transcultural da BCCS para o idioma português, validação de conteúdo e análise da consistência interna. Métodos: Envolveu cinco etapas: (1) tradução; (2) retrotradução; (3) revisão técnica e avaliação das equivalências semântica e conceitual (4) validação de conteúdo por profissionais da área - juízes; (5) avaliação do instrumento por uma amostra de estudantes do sexo feminino. Resultados: O instrumento foi traduzido e adaptado para o idioma português. Foram realizadas algumas adaptações de palavras, expressões e conjugação verbal. Demonstrou ser de fácil compreensão (valores médios superiores a 4,22 - valor máximo 5,0) e excelente concordância (alfa de Cronbach: 0,80 a 0,95). Os construtos verificação objetiva, segurança sobre o corpo, consequências de não checar e controle da dieta e do peso foram identificados pelos especialistas, e o nível de concordância correspondeu a 48,6\%. Conclusão: A escala encontra-se traduzida e adaptada para o idioma português, demonstrando resultados satisfatórios no processo de tradução, adaptação transcultural e análise de consistência interna. São necessárias ainda análises de validade externa, equivalência de mensuração e reprodutibilidade.

Kachani AT, et al. / Rev Psiq Clín. 2011;38(1):13-18

Palavras-chave: Tradução, adaptação transcultural, validade dos testes, transtornos da alimentação, checagem do corpo, cognição.

\begin{abstract}
Background: The Body Checking Cognitions Scale (BCCS) measures cognitions associated with weight checking, which is a medically relevant common behavior among patients with eating disorders (ED). Objective: To translate and cross-culturally adapt BCCS into Brazilian Portuguese, to validate its content and analyze it for internal consistency. Methods: The study included five steps: (1) translation; (2) back translation; (3) technical review and assessment of semantic and conceptual equivalences; (4) content validation by experts - arbiters; and (5) instrument evaluation in a sample of female students. Results: The instrument was translated and adapted into Brazilian Portuguese. Some adjustments were made of words, phrases and verb conjugation. The version was found to be easily understandable (mean values higher than 4.22; maximum value: 5.0 ) and showed excellent concordance (Cronbach's $\alpha$ : 0.80 to 0.95 ). The constructs (objective verification, reassurance, safety beliefs and body control) were identified by experts and the level of agreement was $48.6 \%$. Discussion: BCCS was translated and adapted into Brazilian Portuguese and showed adequate translation, cross-cultural adaptation, and internal consistency. BCCS external validation, measurement equivalence and reproducibility analyses are still required.
\end{abstract}

Kachani AT, et al. / Rev Psiq Clín. 2011;38(1):13-18

Keywords: Translate, cross-cultural adaptation, validity, scales, eating disorder, body checking, cognitions.

\section{Introdução}

Os transtornos alimentares (TA) são síndromes comportamentais cujos critérios diagnósticos têm sido amplamente estudados nos últimos 30 anos. Os principais transtornos alimentares - anorexia nervosa, bulimia nervosa e transtorno alimentar não especificado - compartilham aspectos psicopatológicos comuns, entre eles a preocupação com o peso e a forma do corpo, bem como o medo de engordar ${ }^{1}$. Essa ideia leva os pacientes a se engajarem em dietas restritivas e a utilizarem métodos inapropriados para alcançar um corpo idealizado. Consequentemente, os pacientes têm um controle considerado patológico do peso corporal, associado a distúrbios da percepção do formato de seus corpos, levando a um comportamento alimentar gravemente perturbado. Costumam julgar a si mesmos e aos outros baseando-se quase exclusivamente em sua aparência física, com a qual se mostram sempre insatisfeitos ${ }^{1,2}$.

A fim de verificar sua forma física, os pacientes costumam checar o corpo repetidamente com atitudes de aferição constante de peso, estudar a si mesmo no espelho ou experimentar roupas para avaliar se estão adequadas ou não, beliscar o corpo, comparar seu corpo com o de outras pessoas, entre outras práticas ${ }^{3}$. Em pacientes com TA esses comportamentos podem levar alguns segundos ou prolongar-se por vários minutos muitas vezes ao $\mathrm{dia}^{4}$. Por outro lado, alguns pacientes têm atitude oposta e evitam ao máximo checar seus corpos. Exemplos 
de evitação de checagem do corpo seriam a recusa absoluta em se pesar, cobrir espelhos da casa, evitar se olhar quando passa por vidros e vestir roupas largas para disfarçar o corpo ${ }^{5}$.

Os referidos comportamentos estão associados ao constructo central da doença: superavaliação do corpo, do peso e do que se ingere ${ }^{4,5}$. Segundo Shafran et al. ${ }^{5}$, mais da metade dos pacientes com TA relata que a checagem afeta seu modo de ser e comer, mas que esse comportamento é alternado com o repúdio ao hábito.

Entre os critérios diagnósticos dos Tas, encontramos o comportamento alimentar inadequado, comportamentos compensatórios, perturbação no modo de vivenciar o corpo e o peso, ausência de menstruação por pelo menos três ciclos, entre outros ${ }^{6}$. A insatisfação e/ou distorção da imagem corporal é frequente nos pacientes com TA, chegando inclusive a ser critério diagnóstico para a doença segundo a CID-107. A imagem corporal é uma das questões mais difíceis de serem tratadas nos TA, e uma insatisfação corporal no fim do tratamento pode predizer um mau prognóstico ${ }^{8,9}$. Dessa forma, a frequente checagem do peso está provavelmente relacionada à etiologia e, certamente, à manutenção dos quadros, sustentando erros cognitivos de idealização do peso e do corpo. Se esse comportamento e suas cognições não forem entendidos e explorados durante o tratamento, podem servir para manter a insatisfação e distorção do componente perceptivo da imagem corporal e o consequente comportamento alimentar restritivo9.

Para conhecer as cognições relacionadas ao fenômeno da checagem do corpo, Mountford et al. criaram a Body Checking Cognitions Scale (BCCS), que se apresenta na forma Likert, com 19 questões 9. Para a avaliação do resultado, o escore é calculado pela soma das respostas, podendo variar de 19 a 95 pontos. Quanto maior a pontuação, mais acionado é o aspecto cognitivo e mais consistentes são as crenças que levarão o indivíduo a checar seu corpo ${ }^{9}$.

$\mathrm{Na}$ validação e reprodutibilidade da BCCS, os autores obtiveram bons resultados, confirmando a existência de quatro fatores que compõem o instrumento: 1) verificação objetiva do tamanho corporal; 2) sensação de segurança e garantia da manutenção do peso e boa forma; 3) consequências de não checar o corpo e 4) checagem corporal para uso do controle da dieta e do peso 9 . A consistência do instrumento e das subescalas foi comprovada com valores de 0,96 , $0,72,0,84$ e 0,79 , respectivamente. Trabalharam com grupos clínicos e não clínicos e observaram por meio da comparação das médias que o instrumento foi capaz de discriminar os grupos $(\mathrm{p}<0,001)$. Além da escala em questão, foi aplicado o Body Checking Questionnaire (BCQ), a fim de analisar a correlação entre as subescalas e grupos. A correlação se mostrou positiva e significativa, com valores superiores a $0,30(\mathrm{p}<0,001)^{9}$. É importante salientar que desde 2006, ano da publicação da BCCS, a escala já foi utilizada em três outros trabalhos publicados: 1) Haase et al. ${ }^{10}$, que procuraram compreender o papel da ansiedade na relação entre cognições ligadas à checagem do corpo e seus consequentes comportamentos, apoiando-se na hipótese de que a ansiedade exigiria maior checagem corporal, que por sua vez aumentaria a ansiedade; 2) Mountford et al. ${ }^{11}$, que procuraram determinar se as cognições relacionadas à checagem do corpo são mais relacionadas ao diagnóstico dos subtipos de TA - conforme pesquisas anteriores - ou à sintomatologia alimentar apresentada na doença. Essa pesquisa se justificou pela importância do transdiagnóstico dos TA, em que o foco seria não o diagnóstico em si, mas os comportamentos e cognições relacionados ao problema; 3) Waller et al. ${ }^{12}$, que objetivaram verificar a relação entre checagem do corpo e personalidade narcísica, ou seja, o quanto a variação de níveis de narcisismo influenciaria os diferentes aspectos da checagem do corpo.

Apesar da observação clínica do fenômeno da checagem do corpo, não existem escalas validadas para o nosso meio que possam avaliar o fenômeno a fim de que ele possa ser mais bem estudado. Sendo assim, o objetivo deste trabalho consiste na tradução, adaptação transcultural, validação de conteúdo e análise da consistência interna da Body Checking Cognitions Scale ${ }^{9}$.

\section{Métodos}

A etapa de tradução e adaptação transcultural foi embasada nos procedimentos apontados por Reichenheim e Moraes ${ }^{13}$ e recentemente aplicada por Conti et al. ${ }^{14,15}$.

\section{Procedimentos}

Foram cumpridas cinco etapas: a primeira referente à tradução do instrumento original em inglês para a língua portuguesa; a segunda referente à retrotradução do instrumento em português para a língua inglesa; na terceira, foram realizadas a revisão técnica e a avaliação das equivalências semântica e conceitual; na quarta etapa, foi executada a validação do conteúdo; e na quinta etapa o instrumento foi avaliado por especialistas e estudantes.

A primeira etapa consistiu na tradução do instrumento original do idioma inglês para o português: duas pesquisadoras experientes e fluentes em ambas as línguas traduziram independentemente a escala e discutiram até chegarem numa versão única. Para a segunda etapa, a versão foi novamente traduzida para o inglês, por um professor nativo de língua inglesa. Na etapa seguinte, foram efetuadas a revisão técnica e a avaliação da equivalência semântica das versões, desenvolvida por dois profissionais (uma psicóloga e uma nutricionista), especialistas na área de adaptação de escalas. Foram feitos ajustes e uma nova versão do instrumento foi elaborada.

Para a quarta etapa, a última versão foi apresentada a nove profissionais especialistas da área de transtornos alimentares (três psiquiatras, cinco nutricionistas, dois psicólogos) para avaliação da validade de conteúdo. Para tanto, foi solicitado a cada especialista que identificasse a qual fator cada item pertencia: Verificação objetiva, Segurança sobre o corpo, Consequências de não checar, Controle da dieta e do peso. Como critério de adequação, utilizou-se a taxa de acerto de pelo menos $80 \%$ para cada questão ${ }^{16}$.

Para avaliação da clareza e do grau de compreensão do instrumento em sua íntegra e de cada item que o compõe, foi solicitado, sequencialmente, aos mesmos profissionais, que respondessem a uma escala verbal-numérica adaptada ${ }^{12,13}$. Dessa forma, responderam à seguinte questão: "Você entendeu o que foi perguntado?". As respostas eram do tipo escala Likert: 0 - Não entendi nada; 1 - Entendi só um pouco; 2 - Entendi mais ou menos; 3 - Entendi quase tudo, mas tive algumas dúvidas; 4 - Entendi quase tudo; 5 - Entendi perfeitamente e não tenho dúvidas. Conforme Conti et al. 14,15 , foi estabelecido que as respostas 4 e 5 seriam consideradas como indicadores de uma compreensão suficiente. Paralelamente, foi solicitado a cada especialista, caso não compreendesse alguma questão ou a linguagem não parecesse adequada, que sugerisse alterações, explicitando as razões. Em posse desses resultados, uma nova versão do instrumento foi elaborada.

Na etapa seguinte, a versão final foi apresentada a uma amostra de 154 estudantes de cinco salas dos cursos de Psicologia e Enfermagem, selecionadas de forma aleatória simples. Todos os participantes eram do sexo feminino, com idade média de 24 anos (dp: 6,4 anos). Todas as estudantes concordaram em responder ao questionário de forma voluntária e assinaram o termo de consentimento. Não ocorreu recusa na participação. Para uma parte do grupo (48 estudantes), o instrumento foi aplicado na apresentação de uma escala verbal-numérica adaptada ${ }^{14,15}$ para avaliar o grau de compreensão de cada questão e do instrumento em sua íntegra, conforme metodologia descrita anteriormente. Para as demais participantes (106 jovens), a versão final do instrumento foi aplicada em sua íntegra, com o intuito de avaliar o grau de consistência interna da escala.

Para as análises estatísticas, foi utilizado o programa SPSS versão 15.0. Foram desenvolvidas a análise descritiva (média, desvios-padrão, valores mínimos e máximos, frequências) e a análise inferencial dos dados por meio do cálculo do coeficiente alfa de Cronbach.

O presente estudo está de acordo com a norma ${ }^{\circ} 196$, de 10/10/1996, do Conselho Nacional de Saúde e foi aprovado pelo Comitê de Ética e Pesquisa do Hospital das Clínicas da Faculdade de Medicina da Universidade de São Paulo - Protocolo: 0029/09. 


\section{Resultados}

\section{Tradução e adaptação transcultural}

$\mathrm{Na}$ tradução, ocorreram adaptações de palavras, expressões e conjugação verbal, sempre com a intenção de aproximar a possibilidade de compreensão do sujeito. Dessa forma, ao longo da escala, a expressão "body checking" foi traduzida por "checar o corpo", uma vez que nos pareceu mais adequada para a fluência da leitura. Em várias questões, pronomes pessoais como " $m y$ " ou "me" foram retirados do texto. Em algumas ocasiões (itens 11, 12, 15 e 17), o pronome " $I$ " foi retirado da frase, pois o pronome pessoal do caso reto é importante na língua inglesa, e esta não possui flexões verbais de pessoa para todos os pronomes como acontece na língua portuguesa.

Na questão 1, "allows me" foi vertido como "me dá uma noção para decidir", expressão idiomática mais popular na língua portuguesa. Na questão 2, o verbo "will reassure" foi trocado por "dará segurança"; na questão 3, foi retirada a palavra "down"; na questão 6, "stops me" foi traduzida como "não me deixa" - mudanças essas motivadas porque na língua portuguesa essas expressões se aproximam melhor do sentido original na língua inglesa. O mesmo aconteceu na questão 8, em que "I have put on" foi trocado por "o quanto engordei". Na questão 10, foi retirado o advérbio de intensidade "so", pois para introduzir o advérbio "tão", respeitando o sentido no qual ele foi usado originalmente, teria que mudar a sintaxe da frase. Na questão 11, os verbos "see" e "going" foram trocados por "saber" e "acumulando", por expressarem melhor, na língua portuguesa, o sentido da afirmação no texto original. Na questão 18, a expressão "more confortable" foi substituída por "melhor", uma vez que o adjetivo confortável, na língua portuguesa, normalmente se refere mais a lugares e objetos, o que tiraria o sentido da frase original. Finalmente, na questão 19, foi trocada a palavra "exercise" por "atividade física", expressão que define melhor o tipo de exercício a ser realizado.

Após anuência dos autores da escala, foram introduzidas consígnias a fim de que as entrevistadas soubessem como proce- der, visto que a escala pretende ser de autopreenchimento. Dessa forma, foi adicionado o comando "Responda, numa escala de 1-5, o quanto estes pensamentos vêm à sua cabeça quando pesa o seu corpo. Pontue 1 para menor frequência e vá aumentando até 5 para frequência máxima”. Optou-se por não introduzir ao comando uma definição para as opções de resposta (p. ex.: $1=$ nunca, 2 = raramente, 3 = algumas vezes, $4=$ frequentemente e 5 = sempre) a fim de que a escala original não fosse muito modificada.

As etapas de tradução, retrotradução e versão final estão descritas nas tabelas 1 e 2 .

\section{Compreensão verbal}

Para os especialistas, as questões demonstraram ser de fácil compreensão, registrando valores médios superiores a 4,1 (dp: 1,36). As questões $3,5,6,8,9,11,13$ e 14 obtiveram pontuação máxima, conforme tabela 3 .

O mesmo grau de compreensão pôde ser registrado pelos estudantes. Foram verificados valores superiores a 3,88 (dp: 1,31) e chegando a 4,81 (dp: 0,57) para as questões 5 e 14. Na questão 9, o valor foi igual a $4,94(\mathrm{dp}: 0,32)$ (Tabela 3$)$.

\section{Validação de conteúdo}

O nível de concordância entre os especialistas, para a escala em sua íntegra, correspondeu a 48,6\%. No constructo 1 , relativo à verificação objetiva, a concordância foi de $33,3 \%$. No constructo 2 , que diz respeito à sensação de segurança e garantia da manutenção do peso e boa forma, correspondeu a 75,2\%. Já no constructo 3 , relacionado às consequências de não checar o corpo, atingiu $31 \%$ de concordância, enquanto no constructo 4 , que se refere à checagem corporal como uma forma de controle da dieta e do peso, o valor correspondeu a $55 \%$.

Tabela 1. Avaliação da equivalência semântica - Tradução da Body Checking Cognitions Scale (São Paulo, 2008)

\begin{tabular}{|c|c|}
\hline Documento original & Versão traduzida \\
\hline Body Checking Cognitions Scale (BCCS) & Escala de Checagem Corporal e Cognições (ECCC) \\
\hline & $\begin{array}{l}\text { Responda, numa escala de 1-5, o quanto estes pensamentos vêm à sua cabeça } \\
\text { quando pesa o seu corpo. Pontue } 1 \text { para menor frequência e vá aumentando até } 5 \\
\text { para frequência máxima. }\end{array}$ \\
\hline 1 - Body checking today allows me to decide how much/little I can eat tomorrow. & Checar o corpo hoje me dá uma noção do quanto eu posso comer. \\
\hline 2 - I think body checking will reassure me about my size. & Checar o corpo me dará segurança a respeito do meu tamanho. \\
\hline $\begin{array}{l}3 \text { - I think body checking will help to calm me down when I feel anxious about my } \\
\text { shape or weight. }\end{array}$ & Checar o corpo me acalma em relação à ansiedade sobre meu peso e aparência. \\
\hline 4 - Body checking helps me to control my weight. & Checar o corpo me ajuda a controlar o peso. \\
\hline 5 - Body checking is a good thing for me to do. & Checar o corpo é uma coisa boa de se fazer. \\
\hline 6 - Body checking stops me from losing control of what I eat. & Checar o corpo não me deixa perder o controle sobre o que eu como. \\
\hline 7 - Body checking makes me feel better. & Checar o corpo me faz sentir melhor. \\
\hline 8 - By body checking I can tell how much weight I have put on. & Ao checar meu corpo, eu sei o quanto engordei. \\
\hline 9 - Body checking helps to confirm what the scales say. & Checar o corpo ajuda a confirma o que as escalas mostram. \\
\hline 10 - It's important for me to keep checking because I often feel so enormous. & É importante para mim continuar checando porque me sinto sempre enorme. \\
\hline 11 - I have to body check to see where the weight is going. & Tenho que checar o corpo para saber onde o peso está se acumulando. \\
\hline $\begin{array}{l}12 \text { - I keep checking in the hope that one day I will be happy with the way a look } \\
\text { like. }\end{array}$ & Continuo checando na esperança de que um dia eu fique feliz com minha aparência \\
\hline 13 - If I stop body checking, my weight will shoot up. & Se eu parar de checar o corpo, meu peso vai aumentar muito. \\
\hline 14 - Body checking is the most accurate way to tell what I look like. & Checar o corpo é a forma mais precisa de eu saber como está minha aparência. \\
\hline 15 - If I resist body checking, I will feel worse. & Se eu resistir a checar meu corpo, vou me sentir pior. \\
\hline 16 - I think checking my body will tell me how I feel. & Checar o corpo vai me dizer como eu me sinto. \\
\hline 17 - I can't remember what I look like if I don't check. & Não consigo me lembrar como eu sou se eu não me checar. \\
\hline 18 - I think body checking will make me more comfortable around other people. & Checar o corpo me fará sentir melhor entre outras pessoas. \\
\hline 19 - Body checking tells me when I need to do more exercise. & Checar o corpo me mostra se eu tenho que fazer mais atividade física. \\
\hline
\end{tabular}


Tabela 2. Avaliação da equivalência semântica - Retrotradução e versão final da Body Checking Cognitions Scale (São Paulo, 2008)

\begin{tabular}{|c|c|}
\hline Versão retrotraduzida & Versão final \\
\hline $\begin{array}{l}\text { Answer, on a scale from } 1 \text { to } 5 \text {, how much these thoughts come to your mind } \\
\text { when you weight your body. Score } 1 \text { for small frequency and keep rising till } 5 \text { for } \\
\text { maximum frequency. }\end{array}$ & $\begin{array}{l}\text { Responda, numa escala de 1-5, } 0 \text { quanto estes pensamentos vêm à sua cabeça } \\
\text { quando pesa o seu corpo. Pontue } 1 \text { para menor frequência e vá aumentando até } 5 \\
\text { para frequência máxima. }\end{array}$ \\
\hline 1 - Body checking today gives me a notion of how much I can eat. & $\begin{array}{l}\text { Checar o corpo hoje me dá uma noção para decidir o quanto eu posso comer } \\
\text { amanhã. }\end{array}$ \\
\hline 2 - Body checking will give security in relation to my size. & Eu penso que checar o corpo me dará segurança a respeito do meu tamanho. \\
\hline $\begin{array}{l}3 \text { - Body checking makes me feel calm in relation to the anxiety about my weight } \\
\text { and my physical appearance. }\end{array}$ & $\begin{array}{l}\text { Eu penso que checar o corpo ajudará a me acalmar quando me sentir ansiosa em } \\
\text { relação a meu peso e aparência. }\end{array}$ \\
\hline 4 - Body checking helps me to control the weight. & Checar o corpo me ajuda a controlar o peso. \\
\hline 5 - Body checking is a good thing to do. & Checar o corpo é uma coisa boa de se fazer. \\
\hline 6 - Body checking stops me from losing control about what I eat. & Checar o corpo não me deixa perder o controle sobre o que eu como. \\
\hline 7 - Body checking makes me fell better. & Checar o corpo me faz sentir melhor. \\
\hline 8 - When checking my body, I know how much I've put on weight. & Ao checar meu corpo, eu sei o quanto engordei. \\
\hline 9 - Body checking helps to confirm what the scales show. & Checar o corpo ajuda a confirmar o que as balanças mostram. \\
\hline 10 - It's important to me continuous checking because I always feel enormous. & $\begin{array}{l}\text { É importante para mim continuar checando o meu corpo porque me sinto sempre } \\
\text { enorme. }\end{array}$ \\
\hline 11 - I have to body check in order to know where the weight is accumulating. & Tenho que checar o corpo para saber onde o peso está se acumulando. \\
\hline $\begin{array}{l}12 \text { - I keep checking in the hope that one day I will be happy about my physical } \\
\text { appearance. }\end{array}$ & Continuo checando na esperança de que um dia eu fique feliz com minha aparência. \\
\hline 13 - If I stop body checking, my weight will increase a lot. & Se eu parar de checar o corpo, meu peso vai aumentar muito. \\
\hline $\begin{array}{l}14 \text { - Body checking is the most precise way for me to know how my physical } \\
\text { appearance is. }\end{array}$ & Checar o corpo é a forma mais precisa de eu saber como está minha aparência. \\
\hline 15 - If I resist body checking, I will feel worse. & Se eu resistir a checar meu corpo, vou me sentir pior. \\
\hline 16 - Body checking will tell me how I feel. & Eu penso que checar o corpo vai me dizer como eu me sinto. \\
\hline 17 - I can't remember the way I am if I don't check myself. & Não consigo me lembrar como eu sou se eu não me checar. \\
\hline 18 - Body checking will make me feel better among other people. & Eu penso que checar o corpo me fará sentir melhor entre outras pessoas. \\
\hline 19 - Body checking shows me if I have to do more physical activity. & Checar o corpo me mostra quanto eu tenho que fazer mais atividade física. \\
\hline
\end{tabular}

Tabela 3. Avaliação dos especialistas e dos estudantes da Escala de Checagem Corporal e Cognições (São Paulo, 2009)

\begin{tabular}{|c|c|c|c|c|}
\hline \multirow[t]{2}{*}{ Questões } & \multicolumn{2}{|c|}{$\begin{array}{l}\text { Especialistas } \\
\qquad(\mathrm{n}=10)\end{array}$} & \multicolumn{2}{|c|}{$\begin{array}{c}\text { Estudantes } \\
(n=154)\end{array}$} \\
\hline & $\begin{array}{c}\text { Grau de } \\
\text { concordância } \\
\text { do conteúdo: } \\
A^{*}, B^{* *}, C^{* * *}, \\
D^{* * * *}(\%)\end{array}$ & $\begin{array}{c}\text { Grau de } \\
\text { compreensão } \\
\text { Média (desvios- } \\
\text { padrão) }\end{array}$ & $\begin{array}{c}\text { Grau de } \\
\text { compreensão } \\
\text { Média (desvios- } \\
\text { padrão) } \\
(\mathrm{n}=48)\end{array}$ & $\begin{array}{c}\text { Consistência } \\
\text { interna } \\
\text { Alpha de } \\
\text { Cronbach } \\
(n=106)\end{array}$ \\
\hline 8 & 27,3 & $5(0)$ & $4,77(0,52)$ & \multirow{6}{*}{0,91} \\
\hline 9 & 54,5 & $5(0)$ & $4,94(0,32)$ & \\
\hline 10 & 18,2 & $4,11(1,36)$ & $4,60(0,74)$ & \\
\hline 11 & 18,2 & $5(0)$ & $4,77(0,52)$ & \\
\hline 13 & 45,5 & $5(0)$ & $4,73(0,64)$ & \\
\hline 19 & 36,4 & $4,78(0,67)$ & $4,73(0,57)$ & \\
\hline 2 & 63,6 & $4,78(0,67)$ & $4,25(1,18)$ & \multirow{4}{*}{0,81} \\
\hline 3 & 72,7 & $5(0)$ & $3,88(1,32)$ & \\
\hline 5 & 63,6 & $5(0)$ & $4,81(0,57)$ & \\
\hline 7 & 100 & $4,78(0,44)$ & $4,50(0,92)$ & \\
\hline 14 & 36,4 & $5(0)$ & $4,81(0,45)$ & \multirow{5}{*}{0,88} \\
\hline 15 & 27,3 & $4,56(0,33)$ & $4,08(1,30)$ & \\
\hline 16 & 27,3 & $4,89(0,33)$ & $4,27(1,07)$ & \\
\hline 17 & 27,3 & $4,67(0,50)$ & $4,46(0,8)$ & \\
\hline 18 & 36,4 & $4,56(0,73)$ & $4,10(1,19)$ & \\
\hline 1 & 54,5 & $4,67(1)$ & $4,44(1,0)$ & \multirow{4}{*}{0,80} \\
\hline 4 & 63,6 & $4,56(0,73)$ & $4,44(1,09)$ & \\
\hline 6 & 36,4 & $5(0)$ & $4,56(1,01)$ & \\
\hline 12 & 45,5 & $4,56(0,73)$ & $4,90(0,37)$ & \\
\hline Total & 48,6 & $4,66(0,22)$ & $4,22(0,78)$ & 0,95 \\
\hline
\end{tabular}

${ }^{*} A$ : verificação objetiva - questões $8,9,10,11,13,19$; ** B: segurança sobre 0 corpo - questões $2,3,5,7 ;{ }^{* * *} \mathrm{C}$ : consequências de não checar - questões $14,15,16,17,18 ;{ }^{* * * *} \mathrm{D}$ : controle da dieta e do peso - questões: 1, 4, 6, 12 .

\section{Consistência interna}

O instrumento demonstrou valores do coeficiente alfa de Cronbach excelentes. Para todos os itens que compõem a escala, o valor correspondeu a 0,95 , conforme observado na tabela 3 . Para as questões que integram o conteúdo "verificação objetiva" $(8,9,10,11,13,19)$, "segurança sobre o corpo" $(2,3,5,7)$, "consequências de não checar" $(14,15,16,17,18)$ e "controle da dieta e do peso" $(1,4,6,12)$, os valores corresponderam a $0,91,0,81,0,88$ e 0,80 , respectivamente.

\section{Discussão}

No processo de tradução, foram necessárias várias adaptações para ter o máximo de aproximação e de equivalência de conteúdo trazido da escala original. A equipe de tradução procurou privilegiar a língua de origem a fim de não se desviar do conteúdo apresentado pela escala na língua original. Em quase todas as questões, verbos, pronomes e expressões verbais foram ajustados, garantindo que os significados conotativo e denotativo fossem respeitados na transferência do significado das palavras entre os dois idiomas. O zelo garante a máxima compreensão do instrumento, cumprindo uma das etapas necessárias na avaliação da equivalência semântica, fase crucial da tradução da escala, pois reflete a correspondência de sentido dos conceitos entre o instrumento original e a versão, propiciando efeito semelhante na população, nas duas culturas ${ }^{13,17}$. Em 2003, Bowden e Fox-Rushby ${ }^{18}$, após revisarem várias traduções de escalas, formularam algumas diretrizes para que se obtenha a máxima proximidade entre escala original e escala traduzida, diretrizes essas que foram detalhadamente seguidas neste trabalho.

No processo de adaptação cultural, foi necessária a inserção da instrução de aplicação, bem como a definição das opções na escala Likert de pontos, visto a escala original não apontar essas informações. Esse procedimento se deu com a anuência dos autores.

A escala revelou ser de fácil compreensão com todos os valores, tanto dos especialistas como estudantes, sempre superior a quatro, 
com exceção de uma única questão, a 3, para as estudantes. Provavelmente pelo fato de os conteúdos apresentados não fazerem parte do repertório vivencial do grupo. Mesmo assim, os valores registrados na avaliação da compreensão verbal apontam o sucesso da tradução da escala.

Observando o grau de concordância dos especialistas, pode-se verificar que não houve total coesão na identificação das questões que compõem os construtos. Esse fato se deve, talvez, à proximidade semântica dos conteúdos que compõem as questões, ou mesmo pela falta de domínio conceitual que envolve um assunto tão pouco explorado.

Já em relação à análise da consistência interna, o instrumento apresentou resultados excelentes $(0,95)$, superando o valor apresentado no estudo original $(0,90)$. Esse mesmo efeito pôde ser observado nas análises dos construtos, com uma variação dos valores de alfa de Cronbach de 0,80 a 0,91, para "controle da dieta e do peso" e "verificação objetiva", respectivamente. Observa-se que a consistência interna do instrumento respondeu adequadamente, preservando a coerência e a integração dos itens que compõem os construtos. Dessa forma, pode-se inferir que a escala identificou os mesmos construtos apontados no estudo original.

Vale salientar que, embora a concordância dos especialistas não tenha sido plena, os valores da análise da consistência interna comprovam a adequação do instrumento para a população brasileira.

Apesar da importância clínica da checagem do peso nos TA, pouco foi estudado no intuito de quantificá-la, de perceber o momento quando o comportamento é iniciado, de avaliar o impacto na restrição alimentar e o papel nas cognições preconceituosas dos pacientes. A BCCS é uma escala que avalia cognições relativas à checagem do corpo e abre mais um campo de estudos na área da imagem corporal. Segundo Cash e Deagle ${ }^{19}$, o distúrbio da imagem corporal é um sintoma nuclear dos TA, caracterizado por uma autoavaliação dos indivíduos que sofrem desse transtorno, influenciada pela experiência com seu peso e forma corporal. Dessa forma, uma escala traduzida e adaptada para o idioma português poderá ser utilizada em estudos futuros com enfoque clínico e epidemiológico.

Sabe-se que o desenvolvimento e a validação de escalas têm sido considerados uma importante área de pesquisa científica, principalmente pela necessidade de avaliar e monitorar o tratamento dos pacientes e, assim, verificar sua eficácia ${ }^{18}$. Paralelamente, as escalas são muito úteis em trabalhos populacionais pela sua facilidade e rapidez de aplicação. Instrumentos com tradução e adaptação cultural validados para o meio em que são aplicados garantem que as informações obtidas sejam fidedignas ao grupo pesquisado, podendo ser aplicados em contextos epidemiológico, clínico, de prevenção e promoção da saúde ${ }^{14}$.

Vale ainda ressaltar que são necessárias pesquisas que comprovem a validade externa do instrumento, bem como para populações específicas, como adolescentes, homens, modelos, entre outros.

Uma limitação dessa pesquisa reside no fato de não termos os dados pessoais das estudantes que participaram deste trabalho, o que restringe a possibilidade de generalizarmos os resultados para a população em geral como um todo. Uma vez que o objetivo principal deste trabalho não foi o estudo das cognições relacionadas à checagem do peso, mas sim a adaptação transcultural e a validação do instrumento, essa limitação não compromete os resultados. Outra limitação diz respeito a não existirem muitos pesquisadores na área de imagem corporal que estejam estudando a checagem do corpo. Poder trocar informações é importante para que se possa fazer comparações e avaliar mais precisamente a escala.

\section{Conclusão}

A BCCS foi traduzida e adaptada para o português, apresentando boa compreensão verbal e concordância entre os itens.
A adaptação transcultural e a equivalência semântica são apenas a primeira etapa do processo de validação de um instrumento em qualquer idioma. Para um uso adequado do instrumento, este deve ter boa validade e confiabilidade, além de boa sensibilidade e especificidade. Este estudo é o primeiro passo rumo à determinação desses fatores com a aplicação da versão em português brasileiro da BCCS em uma amostra de pacientes com transtornos alimentares.

\section{Referências}

1. Claudino AM, Borges MBF. Critérios diagnósticos para os transtornos alimentares: conceitos em evolução. Rev Bras Psiquiatr. 2002;24(Supl III):7-12.

2. Saikali CJ, Soubhia CS, Scalfaro BM, Cordás TA. Imagem corporal nos transtornos alimentares. Rev Psiquiatr Clín. 2004;31(4):164-6.

3. Rosen JC. Body image assessment and treatment in controlled studies of eating disorders. Int J Eat Disord. 1997;20:331.

4. Fairburn CG, Cooper Z, Shafran R. Cognitive behaviour therapy for eating disorders: a "transdiagnostic" theory and treatment. Behav Res Ther. 2003;41:509-28.

5. Shafran R, Fairburn CG, Robinson P, Lask B. Body checking and its avoidance in eating disorders. Int J Eat Disord. 2004;35:93-101.

6. Cordás TA. Transtornos alimentares: classificação e diagnóstico. Rev Psiquiatr Clín. 2004;31(4):154-7.

7. Organização Mundial da Saúde (OMS). Classificação dos transtornos mentais e de comportamento da CID-10. Porto Alegre: Artes Médicas; 1993, p. 175-6.

8. Delinsky SS, Wilson GT. Mirror exposure for the treatment of body image disturbance. Int J Eat Disord. 2006;39(2):108-16.

9. Mountford V, Haase AM, Waller G. Body checking in the eating disorders: associations between cognitions and behaviors. Int J Eat Disord. 2006;39:708-15.

10. Haase AM, Mountford V, Waller G. Understanding the link between body checking cognitions and behaviors: the role of social physique anxiety. Int J Eat Disord. 2007;40:241-6.

11. Mountford V, Haase Am, Waller G. Is body checking in the eating disorders more closely related to diagnosis or to symptom presentation? Behav Res Ther. 2007;45:2704-11.

12. Waller G, Sines J, Meyer C, Mountford V. Body checking in the eating disorders: association with narcissistic characteristics. Eat Behav. 2008;9:163-9.

13. Reichenheim ME, Moraes CL. Operacionalização de adaptação transcultural de instrumentos de aferição usados em epidemiologia. Rev Saude Publica. 2007;41(4):665-73.

14. Conti MA, Slater B, Latorre MRDO. Validação e reprodutibilidade da Escala de Evaluación de Insatisfación Corporal para Adolescentes. Rev Saude Publica. 2009;43(3):515-24.

15. Conti MA, Latorre MRDO, Hearst N, Segurado A. Translation into Portuguese, validation and reability of the Body Area Scale for Adolescents. Cad Saude Publica. [no prelo]

16. Pasquali L. Princípios de elaboração de escalas psicológicas. In: Gorenstein C, Andrade LHSG, Zuardi AW, editores. Escalas de avaliação clínica em psiquiatria e psicofarmacologia. São Paulo: Lemos; 2000, p.15-21.

17. Widyanto L, McMurran M. The psychometric properties of the internet addiction test. Cyberpsychol Behav. 2004;7(4):443-50.

18. Bowden A, Fox-Rushby JA. A systematic and critical review of the process of translation and adaptation of generic health-related quality of life measures in Africa, Asia, Eastern Europe, the Middle East, South America. Soc Sci Med. 2003;57:1289-306.

19. Cash TF, Deagle EA. The nature and extend of body image disturbances in anorexia nervosa and bulimia: a meta-analisys. Int J Eat Dis. 1997;22:107-25.

20. Jorge RTB, Sabino Neto M, Natour J, Veiga DF, Jones D, Ferreira LM. Brazilian version of the Body Dysmorphic Disorder Examination. São Paulo Med J. 2008;126(2):87-95. 
Anexo 1. Escala de Checagem Corporal e Cognições (Body Checking Cognitions Scale-BCCS)

Responda, numa escala de 1-5, 0 quanto estes pensamentos vêm à sua cabeça quando pesa o seu corpo. Pontue 1 para menor frequência e vá aumentando até 5 para frequência máxima.

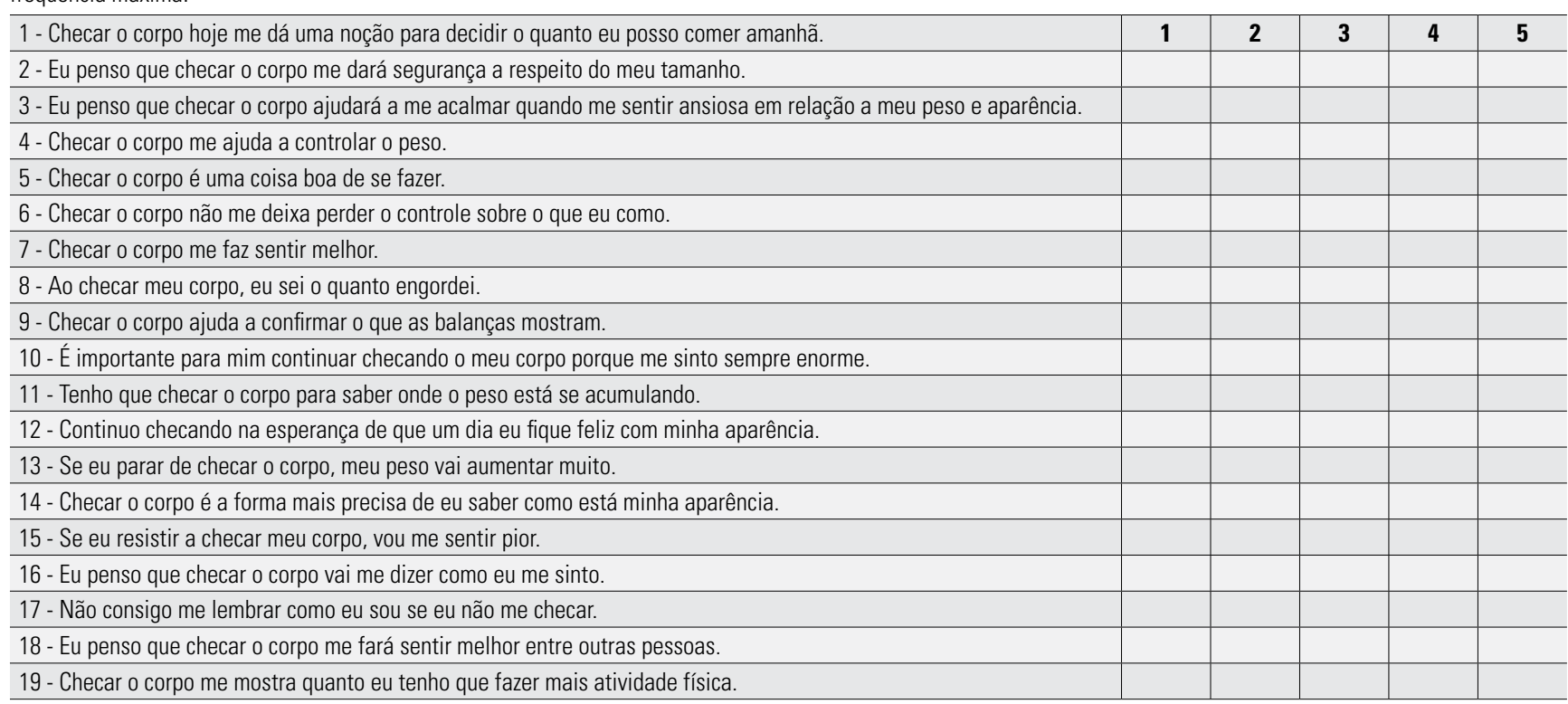

